\title{
Bayesian comparison of interacting modified holographic Ricci dark energy scenarios
}

\author{
Antonella Cid $^{1,2, a}$, Carlos Rodriguez-Benites ${ }^{3, \mathrm{~b}}$, Mauricio Cataldo ${ }^{1, \mathrm{c}}$, Gonzalo Casanova ${ }^{4, \mathrm{~d}}$ \\ ${ }^{1}$ Departamento de Física, Universidad del Bío-Bío, Casilla 5-C, Concepción, Chile \\ 2 Centro de Ciencias Exactas, Universidad del Bío-Bío, Casilla 447, Chillán, Chile \\ ${ }^{3}$ Escuela Central de Posgrado, Universidad Nacional de Ingeniería, Lima, Peru \\ ${ }^{4}$ Departamento de Física, Universidad de Concepción, Casilla 160-C, Concepción, Chile
}

Received: 20 May 2020 / Accepted: 6 January 2021 / Published online: 15 January 2021

(C) The Author(s) 2021

\begin{abstract}
We perform a Bayesian model selection analysis for interacting scenarios of dark matter and modified holographic Ricci dark energy (MHRDE) with linear interacting terms. We use a combination of some of the latest cosmological data such as type Ia supernovae, cosmic chronometers, the local value of the Hubble constant, baryon acoustic oscillations measurements and cosmic microwave background through the angular scale of the sound horizon at last scattering. We find moderate/strong evidence against all the MHRDE interacting scenarios studied with respect to $\Lambda \mathrm{CDM}$ when the full joint analysis is considered.
\end{abstract}

\section{Introduction}

It is well known that our universe is currently in a phase of accelerated expansion [1]. This acceleration is driven by the so-called dark energy, which in the standard cosmological model is represented by a cosmological constant $\Lambda$. The standard cosmological model or $\Lambda \mathrm{CDM}$ provides a good explanation for the current acceleration but it has some drawbacks, the cosmological constant problem [2,3], the coincidence problem $[4,5,12]$ and the tension in the values obtained for the Hubble parameter from local measurements and inferred from Planck's data [13-15].

Over the last twenty years, many dark energy (DE) models have been proposed in order to explain the observed current acceleration of the universe (see Refs. [16-19] for reviews on this topic). In particular, holographic dark energy (HDE) models are founded on the holographic principle [20]. In this

\footnotetext{
a e-mail: acidm@ubiobio.cl (corresponding author)

be-mail: carlos.rodriguez.b@uni.pe

c e-mail: mcataldo@ubiobio.cl

de-mail: gonzalocasanova@udec.cl
}

context, based in the Bekenstein-Hawking entropy bound of black holes thermodynamics [21,25], in Ref. [27] it was postulated that the energy contained in a region of size $L$ must not exceed the mass of a black hole of the same size, i.e., in terms of the energy density $\rho, \rho \leq L^{-2}$. In a cosmological setup, the largest scale $L$ allowed is the one saturating this inequality. Several dark energy models motivated by the holographic principle have been explored. Among these, it has been shown that by choosing the scale $L$ as the Hubble length or the size of the particle horizon it is not possible to obtain accelerated expansion [28,29]. In Ref. [29] it was proposed a model where the scale $L$ is given by the size of the future event horizon leading to a successful HDE model but, this model has been criticized based on causality issues [30]. In Ref. [31], the holographic Ricci dark energy (HRDE) model was proposed, where the dark energy density is postulated as proportional to the Ricci scalar and this model does not present causality problems. Subsequently, in Ref. [32] it was noticed that the Jeans length of the perturbations sets a causal connection scale given by the Ricci scalar, providing the HRDE model with a physical motivation. There are many studies on these kind of models, e.g., see Refs. [33-41]. In Ref. [42] a generalization or modified holographic Ricci dark energy model was proposed, where the dark energy density is given by

$\rho_{x}=3\left(\alpha H^{2}+\beta \dot{H}\right)$

for $\alpha$ and $\beta$ constants. Notice that for $\beta=2 \alpha$, the HRDE model is recovered in a flat scenario. Furthermore, in Ref. [43] it was pointed out that the scale $L$ leading to (1) is a natural extension of the Hubble length considered in the original HDE model. This model has been widely studied in the literature, see Refs. [43-47]. 
In recent years, several entropy formalisms have been used in proposing HDE models, such as: Tsallis [48], Renyi [49], Sharma-Mittal [50] and Barrow [51]. These new models are based in generalized entropies, instead of the BekensteinHawking entropy $[21,25]$, applied to a cosmological context.

On the other hand, interacting scenarios, where dark matter and dark energy are coupled, have become very appealing because, in the last years, it has been claimed that they can alleviate the $H_{0}$ tension (see Refs. [6-11]). In this sense, interacting HDE models have been extensively studied, originally with the aim of find an accelerating scenario with $L=H$ [12]. Over the years, many interacting holographic scenarios have been studied in different contexts, see for example Refs. [52-71].

In particular, scenarios with linear interaction, where the components are dark matter $\rho_{m}$ and holographic dark energy $\rho_{x}$, with interaction terms of the type $Q \propto H \rho_{m}, Q \propto H \rho_{x}$ and $Q \propto H\left(\rho_{m}+\rho_{x}\right)$ are studied in $[53,59,64]$. Interacting terms proportional to the dark energy densities and/or its derivatives in the context of modified holographic dark energy were studied in Refs. [54,56-58]. Likewise, there are several models of non-linear interaction for dark matter and holographic dark energy, e.g., interacting terms $Q \propto$ $H\left(\frac{\rho_{x}^{2}}{\rho_{m}+\rho_{x}}\right), Q \propto\left(\frac{\rho_{m} \rho_{x}}{H}\right)$ and $Q \propto H\left(\frac{\rho_{m} \rho_{x}}{\rho_{m}+\rho_{x}}\right)$ have been studied in Refs. [62,64,65,70], respectively.

In Refs. [36,40,46,53,70-73] the performance of holographic dark energy models in fitting the data has been compared with the $\Lambda \mathrm{CDM}$ model, where several criteria has been used, $\chi^{2} /$ dof, AIC and BIC [74] and Bayesian evidence. In this sense, Bayesian model selection through the Bayesian evidence is a more powerful statistical tool in comparing the performance of cosmological models in light of the more recent available data and it has been widely used in cosmology [75-79]. In particular, in Ref. [80] inconclusive evidence was found in studying a class of interacting models when compared to $\triangle \mathrm{CDM}$ and considering background data.

The aim of this paper is to analyze the observational viability of interacting scenarios considering modified holographic Ricci dark energy. To asses the models' viability we perform a Bayesian model selection analysis and compare interacting modified holographic Ricci dark energy (IMHRDE) scenarios with the $\Lambda$ CDM model in light of background data such as supernovae type Ia, cosmic chronometers, the local value of the Hubble constant, baryon acoustic oscillations and the angular scale of the sound horizon at the last scattering. The paper is organized as follows. In Sect. 2 we find analytical solutions for the studied scenarios and describe the kinematics of these models. In Sect. 3 we describe the data used and the methodology. In Sect. 4 we discuss the main results and in Sect. 5 we present the final remarks.

\section{The interacting modified holographic dark energy model}

Let us consider a flat, homogeneous and isotropic universe in the framework of General Relativity, the spatially flat Friedmann-Lemaître-Robertson-Walker (FLRW) metric. The Friedmann's equation in this context is written as

$3 H^{2}=\rho$,

where $\rho$ is the total energy density and $8 \pi G=c=1$ is assumed. On the other hand, from the conservation of the total energy-momentum tensor we have

$\dot{\rho}+3 H(\rho+p)=0$,

where $p$ is the total pressure. A realistic cosmological scenario contains baryons $(b)$, radiation $(r)$, cold dark matter $(c)$ and a dark energy $(x)$ components, in this work this last component is assumed to be given by holographic dark energy. In this context we consider the Friedmann equation (2) and the conservation equation (3) assuming $\rho=\rho_{b}+\rho_{r}+\rho_{c}+\rho_{x}$ and $p=p_{b}+p_{r}+p_{c}+p_{x}$. From here on and for the sake of simplicity we define $\rho_{d}=\rho_{c}+\rho_{x}$. In addition, a barotropic equation of state is considered for all the components, $p_{i}=\omega_{i} \rho_{i}$ with $\omega_{b}=0, \omega_{r}=1 / 3, \omega_{c}=0$ and $\omega_{x}=\omega$ as a state function. Furthermore, by including a phenomenological interaction in the dark sector $\Gamma$, we separate the conservation equation (3) into the following equations

$$
\begin{aligned}
\rho_{b}^{\prime}+\rho_{b} & =0, \\
\rho_{r}^{\prime}+\frac{4}{3} \rho_{r} & =0, \\
\rho_{c}^{\prime}+\rho_{c} & =-\Gamma, \\
\rho_{x}^{\prime}+(1+\omega) \rho_{x} & =\Gamma,
\end{aligned}
$$

where by convenience we use the change of variable $\eta=$ $3 \ln a$ and define ()$^{\prime}=d / d \eta$. Note that $\Gamma>0$ indicates an energy transfer from cold dark matter to dark energy and $\Gamma<0$ indicates the opposite. From Eqs. (1) and (2) we can easily notice that

$\rho_{x}=\alpha \rho+\frac{3 \beta}{2} \rho^{\prime}$

By deriving Eq. (8) and replacing $\rho_{x}^{\prime}=\rho_{d}^{\prime}-\rho_{c}^{\prime}, \rho_{c}^{\prime}$ from Eq. (6), $\rho_{c}=\rho_{d}-\rho_{x}, \rho_{x}$ from Eq. (8), $\rho=\rho_{b}+\rho_{r}+\rho_{d}$, $\rho_{b}^{\prime \prime}=\rho_{b}, \rho_{r}^{\prime \prime}=\frac{16}{9} \rho_{r}$ and $\rho_{r}$ by the solution of Eq. (5), in this order, we obtain a second order differential equation for the energy density of the dark sector $\rho_{d}$,

$$
\begin{gathered}
\frac{3 \beta}{2} \rho_{d}^{\prime \prime}+\left(\alpha+\frac{3 \beta}{2}-1\right) \rho_{d}^{\prime}+(\alpha-1) \rho_{d} \\
+\frac{1}{3}(2 \beta-\alpha) \rho_{r} 0 a^{-4}=\Gamma,
\end{gathered}
$$


where $\rho_{r 0}$ is the integration constant from Eq. (5). For a given interaction $\Gamma=\Gamma\left(\rho_{d}, \rho_{d}^{\prime}, \rho, \rho^{\prime}\right)$, we can analytically solve Eq. (9) to find the energy density $\rho_{d}$, and consequently the energy densities $\rho_{x}$ and $\rho_{c}$ through Eq. (8).

In this work we study the general linear interaction,

$\Gamma=\alpha_{1} \rho_{c}+\beta_{1} \rho_{x}$,

which includes four different types of interaction, $\alpha_{1}=0$, $\beta_{1}=0, \alpha_{1}=\beta_{1}$ and $\alpha_{1} \neq \beta_{1}$. Notice that it is possible to describe all these interactions with terms proportional to $\rho_{d}$, $\rho_{d}^{\prime}, \rho$ and $\rho^{\prime}$. Then, we can rewrite Eq. (9) as

$\rho_{d}^{\prime \prime}+b_{1} \rho_{d}^{\prime}+b_{2} \rho_{d}+b_{3} a^{-3}+b_{4} a^{-4}=0$,

including the four interaction types of our interest, where the constants are defined as

$b_{1}=1+\alpha_{1}-\beta_{1}-2(1-\alpha) / 3 \beta$,

$b_{2}=\frac{2}{3 \beta}\left(\alpha\left(1-\beta_{1}+\alpha_{1}\right)-1-\alpha_{1}\right)$,

$b_{3}=\Omega_{b 0}\left(\beta_{1}-\alpha_{1}\right)(1-2 \alpha / 3 \beta)$,

$b_{4}=\frac{2 \Omega_{r 0}}{3 \beta}\left((2 \beta-\alpha) / 3-\left(\beta_{1}-\alpha_{1}\right)(\alpha-2 \beta)\right)$,

and $\Omega_{b 0}$ and $\Omega_{r 0}$ are the density parameters (i.e. $\Omega_{i 0}=$ $\rho_{i 0} / 3 H_{0}^{2}$ with $i=\{b, r\}$ for baryons, the radiation and $H_{0}$ is the Hubble parameter). The general solution of equation (11) has the form

$\rho_{d}(a)=3 H_{0}^{2}\left(\frac{A}{a^{3}}+\frac{B}{a^{4}}+C_{1} a^{3 \lambda_{1}}+C_{2} a^{3 \lambda_{2}}\right)$,

where the integration constants are given by

$$
\begin{aligned}
C_{1}= & \frac{A\left(1+\lambda_{2}\right)}{\left(\lambda_{1}-\lambda_{2}\right)}+\frac{B\left(4+3 \lambda_{2}\right)}{3\left(\lambda_{1}-\lambda_{2}\right)}+\frac{2\left(\Omega_{x 0}-\alpha\right)}{3 \beta\left(\lambda_{1}-\lambda_{2}\right)} \\
& +\frac{3 \Omega_{b 0}+4 \Omega_{r 0}-3 \lambda_{2}\left(\Omega_{c 0}+\Omega_{x 0}\right)}{3\left(\lambda_{1}-\lambda_{2}\right)}, \\
C_{2}= & -A-B+\Omega_{c 0}+\Omega_{x 0}-C_{1},
\end{aligned}
$$

and $\Omega_{c 0}, \Omega_{x 0}$ are the density parameters for the cold dark matter and the MHRDE, respectively. The coefficients in (12) are given by,

$$
A=\frac{b_{3}}{b_{1}-b_{2}-1}, \quad B=\frac{9 b_{4}}{12 b_{1}-9 b_{2}-16},
$$

and $\lambda_{1,2}=-\frac{1}{2}\left(b_{1} \pm \sqrt{b_{1}^{2}-4 b_{2}}\right)$.

Therefore, the Hubble expansion rate can be written as:

$$
H(a)^{2}=H_{0}^{2}\left(\frac{\bar{A}}{a^{3}}+\frac{\bar{B}}{a^{4}}+C_{1} a^{3 \lambda_{1}}+C_{2} a^{3 \lambda_{2}}\right),
$$

where $\bar{A}=A+\Omega_{b 0}, \bar{B}=B+\Omega_{r 0}, \Omega_{b 0}+\Omega_{r 0}+\Omega_{c 0}+\Omega_{x 0}=$ 1 and the radiation term includes the contribution of photons, $\Omega_{\gamma 0}$, and massless neutrinos, $\Omega_{\nu 0}$.

Notice that, without an interacting term $\left(\alpha_{1}=\beta_{1}=0\right)$, a HRDE model, $\rho_{x}=\alpha\left(2 H^{2}+\dot{H}\right)$, is recovered from (16) for $\alpha=2 \beta, b_{3}=b_{4}=0$ and $A=B=0$. Likewise, a MHRDE model, $\rho_{x}=3\left(\alpha H^{2}+\beta \dot{H}\right)$, is recovered from (16) for $b_{3}=0$ and $A=0$.

On the other hand, using Eqs. (8), (10) and (12) into (7), we obtain an expression for the variable state parameter,

$\omega(a)=\frac{D_{1} a^{-3}+D_{2} a^{-4}+D_{3} a^{3 \lambda_{1}}+D_{4} a^{3 \lambda_{2}}}{\tilde{A} a^{-3}+\tilde{B} a^{-4}+\tilde{C}_{1} a^{3 \lambda_{1}}+\tilde{C}_{2} a^{3 \lambda_{2}}}$,

where $\tilde{A}=(2 \alpha-3 \beta) \bar{A}, \tilde{B}=2(\alpha-2 \beta) \bar{B}, \tilde{C}_{1}=C_{1}\left(3 \beta \lambda_{1}+\right.$ $2 \alpha), \tilde{C}_{2}=C_{2}\left(3 \beta \lambda_{2}+2 \alpha\right), D_{1}=2 \alpha_{1} A+\left(\beta_{1}-\alpha_{1}\right) \tilde{A}$, $D_{2}=2 \alpha_{1} B+\left(1 / 3-\alpha_{1}+\beta_{1}\right) \tilde{B}, D_{3}=-2 C_{1}\left(1+\lambda_{1}\right)$ and $D_{4}=-2 C_{2}\left(1+\lambda_{2}\right)$. In the limit to the future $(a \rightarrow \infty)$, the expression (17) becomes $\omega \rightarrow \frac{-2\left(1+\lambda_{2}\right)}{3 \beta \lambda_{2}+2 \alpha}$ for $\lambda_{2}>\lambda_{1}>-1$, which could assume positive or negative values depending on the interacting and holographic parameters.

In addition, from $\rho_{c}=\rho_{d}-\rho_{x},(8)$ and (12), the coincidence parameter $\left(r=\rho_{c} / \rho_{x}\right)$ becomes,

$r(a)=\frac{\hat{A} a^{-3}+\hat{B} a^{-4}+\hat{C}_{1} a^{3 \lambda_{1}}+\hat{C}_{2} a^{3 \lambda_{2}}}{\tilde{A} a^{-3}+\tilde{B} a^{-4}+\tilde{C}_{1} a^{3 \lambda_{1}}+\tilde{C}_{2} a^{3 \lambda_{2}}}$

where $\hat{X}=2 X-\tilde{X}$ for $X=A, B, C_{1}, C_{2}$. Therefore, the asymptotic limit of $r(a)$ as $a \rightarrow \infty$ for $\lambda_{2}>\lambda_{1}>-1$ becomes

$r_{\infty} \rightarrow \frac{2}{3 \beta \lambda_{2}+2 \alpha}-1$,

a constant depending on the interacting and holographic parameters.

Furthermore, from Eq. (12) we can obtain the deceleration parameter $q=-\left(1+\frac{3 \rho^{\prime}}{2 \rho}\right)$ as

$q(a)=-1+\frac{3}{2} \frac{\frac{\bar{A}}{a^{3}}+\frac{4}{3} \frac{\bar{B}}{a^{4}}-C_{1} \lambda_{1} a^{3 \lambda_{1}}-C_{2} \lambda_{2} a^{3 \lambda_{2}}}{\frac{\bar{A}}{a^{3}}+\frac{\bar{B}}{a^{4}}+C_{1} a^{3 \lambda_{1}}+C_{2} a^{3 \lambda_{2}}}$.

Notice that in the asymptotic limit $a \rightarrow \infty$ we get $q \rightarrow$ $-1-\frac{3}{2} \lambda_{2}$ for $\lambda_{2}>\lambda_{1}>-1$.

\section{Observational analysis}

In the observational analysis we use data such as supernovae type Ia from the Pantheon Sample [81], cosmic chronometers, obtained through the differential age method and reported in Ref. [82], the local value of the Hubble constant [83], baryon acoustic oscillations (from 6dFGS [84], 
SDSS-MGS [85], eBOSS [86,87] and BOSS DR12 [88]), and the angular scale of the sound horizon at the last scattering [89]. In the following, we briefly present these datasets.

\subsection{Supernovae type Ia}

We use the most up to date compilation of supernovae type Ia (SNe Ia), the Pantheon Sample, containing a set of 1048 spectroscopically confirmed SNe Ia [81] ranging from redshift 0.01 to 2.3, along with a covariance matrix (including statistical and systematic errors). The Pantheon catalog contains measurements of peak magnitudes in the B-band's rest frame, $m_{B}$, which are related to the distance modulus as $\mu=m_{B}+M_{B}$, where $M_{B}$ is a nuisance parameter corresponding to the absolute B-band magnitude of a fiducial SN Ia. In our analysis we theoretically compute the distance modulus at a given redshift as

$\mu(z)=5 \log d_{L}(z)+25$,

where $d_{L}$ is the luminosity distance in units of Mpc,

$d_{L}=(1+z) \int_{0}^{z} \frac{H_{0} d z^{\prime}}{H\left(z^{\prime}\right)}$,

and $H_{0}$ is the Hubble parameter.

\subsection{Cosmic chronometers}

We use 24 cosmic chronometers (CC) obtained through the differential age method (see Table 4 in [82]) by taking the relative age of passively evolving galaxies with respect to the redshift [90]. This procedure provides cosmologicalindependent direct measurements of the expansion history of the universe up to redshift 1.2 [91]. In our analysis, the theoretical value of the Hubble expansion rate is given by equation (16).

\subsection{Local determination of the Hubble constant}

We consider the most recent value of the Hubble constant deduced by the SHOES team (R19) using a local distance ladder method based on Cepheids, $h=0.7402 \pm 0.0142$ [83]. This value is in $4.4 \sigma$ tension with the prediction of data from Planck, $h=0.6736 \pm 0.0054$ [92].

\subsection{Baryon acoustic oscillation (BAO)}

The isotropic measurements of the $\mathrm{BAO}$ signal are given in terms of the dimensionless ratio

$d_{z}(z)=D_{V}(z) / r_{s}\left(z_{d}\right)$ where $D_{V}$ is a combination of the line-of-sight and transverse distance scales defined in Ref. [93], $z_{d}$ is the redshift at the drag epoch and $r_{s}(z)$ is the comoving size of the sound horizon, where $D_{V}$ and $r_{s}$ are defined by

$$
\begin{aligned}
D_{V}(z) & =\left((1+z)^{2} D_{A}(z)^{2} \frac{c z}{H(z)}\right)^{1 / 3} \text { and } \\
r_{S}(z) & =\int_{z}^{\infty} \frac{c_{s} d z}{H(z)}
\end{aligned}
$$

respectively, with $c$ the speed of light, $D_{A}(z)=\frac{c}{(1+z)} \int_{0}^{z} \frac{d z}{H(z)}$ the angular diameter distance, $c_{s}=\frac{c}{\sqrt{3(1+R)}}$ being the sound speed of the photon-baryon fluid and $R=\frac{3 \Omega_{b}}{4 \Omega_{\gamma}(1+z)}$ [94].

We use isotropic BAO measurements from 6dFGS [84], MGS [85] and eBOSS [86].

Furthermore, we use the anisotropic BAO measurements from BOSS DR12 [88], which are defined in terms of $D_{A}$ and $D_{H}=c / H(z)$, as shown in Table 2 of Ref. [95]. We use these data along with the corresponding covariance matrix in Ref. [95].

\subsection{Cosmic microwave background (CMB)}

The only contribution of CMB data we consider in this work is the angular scale of the sound horizon at the last scattering:

$\ell_{a}=\frac{\pi\left(1+z_{*}\right) D_{A}\left(z_{*}\right)}{r_{S}\left(z_{*}\right)}$

where the comoving size of the sound horizon is evaluated at $z_{*}=1089.80$, according with Planck's 2018 results [92]. We compare the value obtained in our study with the one reported by the Planck collaboration in 2015, $\ell_{a}=301.63 \pm 0.15$ [89].

\subsection{Bayesian model selection}

The Bayesian inference (based on the Bayes' theorem) is a robust statistical technique for parameter estimation and model selection widely used in the study of cosmological scenarios $[74,80]$. The Bayes' theorem relates the posterior probability $P$ for a set of parameters $\Theta$, given the data $\mathcal{D}$, described by a model $\mathcal{M}$,

$P(\Theta \mid \mathcal{D}, \mathcal{M})=\frac{\mathcal{L}(\mathcal{D} \mid \Theta, \mathcal{M}) \mathcal{P}(\Theta \mid \mathcal{M})}{\mathcal{E}(\mathcal{D} \mid \mathcal{M})}$

where $\mathcal{L}, \mathcal{P}$ and $\mathcal{E}$ are the likelihood, prior and evidence, respectively.

In comparing the performance of two different models given a dataset, we use the Bayes' factor defined as the ratio of the evidences of models $M_{1}$ and $M_{2}$ as:

$B_{12}=\mathcal{E}_{1} / \mathcal{E}_{2}$ 
where the evidence is obtained integrating Eq. (26) over the space of parameters. If the models $M_{1}$ and $M_{2}$ have the same prior probability, then the Bayes factor gives the posterior odds of the two models.

To compare the studied models with the $\Lambda \mathrm{CDM}$ model, we use a conservative version of the Jeffreys' scale defined in Ref. [96]. This scale gives us an empirical measure for interpreting the strength of the evidence in comparing two competing models. The Jeffreys' scale interprets the evidence as follows, inconclusive if $\left|\ln B_{12}\right|<1$, weak if $1 \leq\left|\ln B_{12}\right|<2.5$, moderate if $2.5 \leq\left|\ln B_{12}\right|<5$ and strong if $\left|\ln B_{12}\right| \geq 5$. In all the cases, the evidence is interpreted as in favor of the tested model if $\ln B_{12}$ is positive or against if negative.

In our work we consider $\Lambda \mathrm{CDM}$ as the reference model, as such, the subscripts in the Bayes' factor (27) will be omitted hereafter.

To compute the evidence and generate the posterior distributions we use the MULTINEST algorithm ${ }^{1}$ [97,98], requiring a global log-evidence tolerance of 0.01 as a convergence criterion and working with a set of 800 live points to improve the accuracy in the estimation of the evidence.

\section{Analysis and results}

We performed a Bayesian comparison analysis of the general interaction model $\Gamma=\alpha_{1} \rho_{c}+\beta_{1} \rho_{x}$ with the $\Lambda$ CDM model in terms of the strength of the evidence according to the Jeffreys' scale. Three different dataset combination are considered in the present work, namely, Pantheon $+\mathrm{CC}$, Pantheon $+\mathrm{CC}+$ $\mathrm{R} 19+\mathrm{BAO}+\mathrm{CMB}$ and Pantheon $+\mathrm{CC}+\mathrm{BAO}+\mathrm{CMB}$. Also, we analyse two different scenarios, the impact of considering a Gaussian prior on the parameter $\omega_{b}=\Omega_{b} h^{2}$ and the impact of including or not the R19 data in the full joint analysis.

In the studied models the prior probability distributions for free parameters are shown in Table 1. We have chosen a uniform prior for parameters such as $\Omega_{c}, \alpha, \beta, \alpha_{1}, \beta_{1}$, $M_{B}$ and $h$, and a Gaussian prior for the parameter $\omega_{b}$. For the parameter $\Omega_{c}$ we choose a conservative uniform prior between 0 and 1 , for the dimensionless Hubble parameter $h$ we adopt a Uniform prior between 0.6 and 0.8 . The priors for the holographic parameters were considered positive and small $[57,60]$, the prior for the interacting parameters are uniform distributions centered in zero and for the Pantheon Sample parameter $M_{B}$, we use a conservative range including the value reported by Scolnic et al. in Ref. [81]. For the analysis including the parameter $\omega_{b}$, we have chosen a Gaussian prior centered in the value reported in Ref. [99].

We expect interacting models mainly affect the late time evolution and not the physics of the primordial uni-

\footnotetext{
${ }^{1}$ https://github.com/JohannesBuchner/MultiNest.
}

Table 1 Prior probability distributions for the models' parameters. For a Gaussian (G) prior we inform $\left(\mu, \sigma^{2}\right)$ and for a uniform (U) prior we inform $(a, b)$ representing $a \leq x \leq b$

\begin{tabular}{lll}
\hline Parameters & Prior & Refs. \\
\hline$h$ & $\mathrm{U}(0.6,0.8)$ & - \\
$\Omega_{c}$ & $\mathrm{U}(0,1)$ & - \\
$\alpha$ & $\mathrm{U}(0,1)$ & {$[57,60]$} \\
$\beta$ & $\mathrm{U}(0,1)$ & {$[57,60]$} \\
$\alpha_{1}$ & $\mathrm{U}(-1,1)$ & {$[74,80]$} \\
$\beta_{1}$ & $\mathrm{U}(-1,1)$ & {$[74,80]$} \\
$M_{B}$ & $\mathrm{U}(-20,-18)$ & {$[81]$} \\
$\omega_{b}$ & $\mathrm{G}(0.02235,0.00033)$ & {$[99]$} \\
\hline
\end{tabular}

verse. In this sense we consider the following constraints: $\Omega_{r}=\Omega_{\gamma}\left(1+\frac{7}{8}\left(\frac{4}{11}\right)^{\frac{4}{3}} N_{\text {eff }}\right)$ with $N_{\text {eff }}=3.046$ [100], and $\Omega_{\gamma} h^{2}=2.469 \times 10^{-5}$ [101]. Moreover, for the redshift at the drag epoch and the last scattering epoch we use Planck's results [92], $z_{d}=1060.01$ and $z_{*}=1089.80$, respectively.

Our main results are summarized in Tables 2 and 3 . We labeled the studied interacting modified holographic Ricci dark energy models (IMHRDE) in the following way, IMHRDE $1,2,3,4$ representing $\alpha_{1}=0, \beta_{1}=0, \alpha_{1}=\beta_{1}$, $\alpha_{1} \neq \beta_{1}$, respectively.

In Tables 2 and 3 we present the mean value and $1 \sigma$ error for the parameters of all the studied models, along with the logarithm of the evidence, the logarithm of the Bayes factor and the interpretation for the strength of the evidence. In Table 2 we notice that by considering Pantheon + R19, we get weak evidence against for each of the studied IMHRDE when compared to $\Lambda \mathrm{CDM}$. Also, in Table 2, the results for the full joint analysis Pantheon $+\mathrm{CC}+\mathrm{R} 19+\mathrm{BAO}+\mathrm{CMB}$ are shown, here we find moderate/strong evidence against for each of the studied IMHRDE models, i.e., including data such as BAO or CMB shift the evidence to a better support for $\Lambda$ CDM. On the other hand, in Table 2 we fix the physical baryon density to $\omega_{b}=0.02235$, as reported in [99], in contrast, in Table 3 we analyse the impact of including the baryon density $\omega_{b}$ as a free parameter with a Gaussian prior. Comparing Tables 2 and 3 we notice that the posterior of the $\omega_{b}$ parameter remains very close to the prior in all analysed cases and the remaining parameters are unaffected inside the $1 \sigma$ region, i.e., the parameter $\omega_{b}$ has a minimal incidence in the results. Furthermore, in Table 3 we show the joint analyses Pantheon $+\mathrm{CC}+\mathrm{R} 19+\mathrm{BAO}+\mathrm{CMB}$ and Pantheon $+\mathrm{CC}+\mathrm{BAO}+\mathrm{CMB}$, in order to revise the incidence of the R19 point in the results. We notice that by including R19 we get more support for the IMHRDE models but Bayesian evidence still favours $\Lambda \mathrm{CDM}$. On the other hand, from our results it could seem the $H_{0}$ tension is alleviated because the IMHRDE models have a higher value for the $h$ parameter (closer to R19) compared with the one obtained 


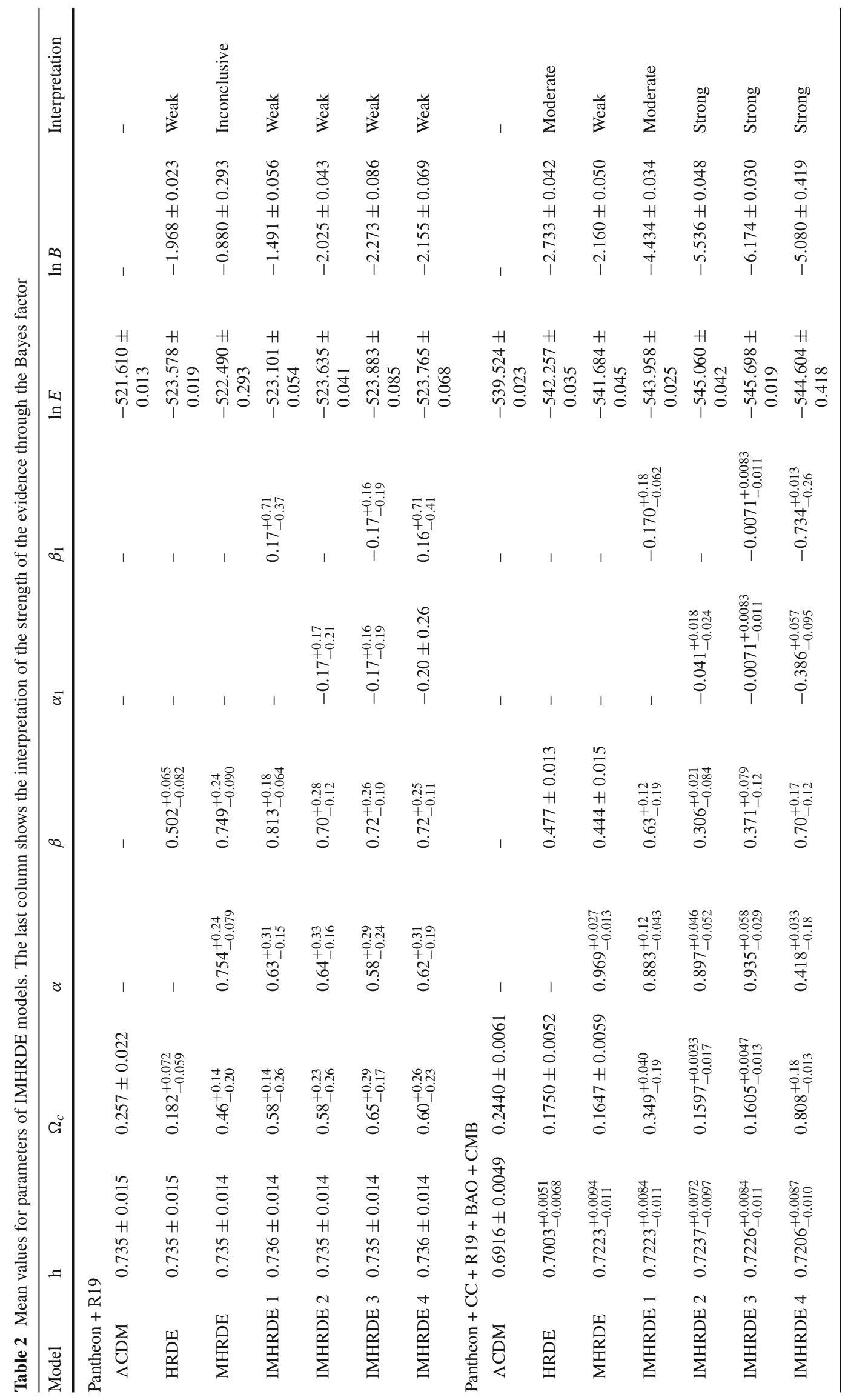




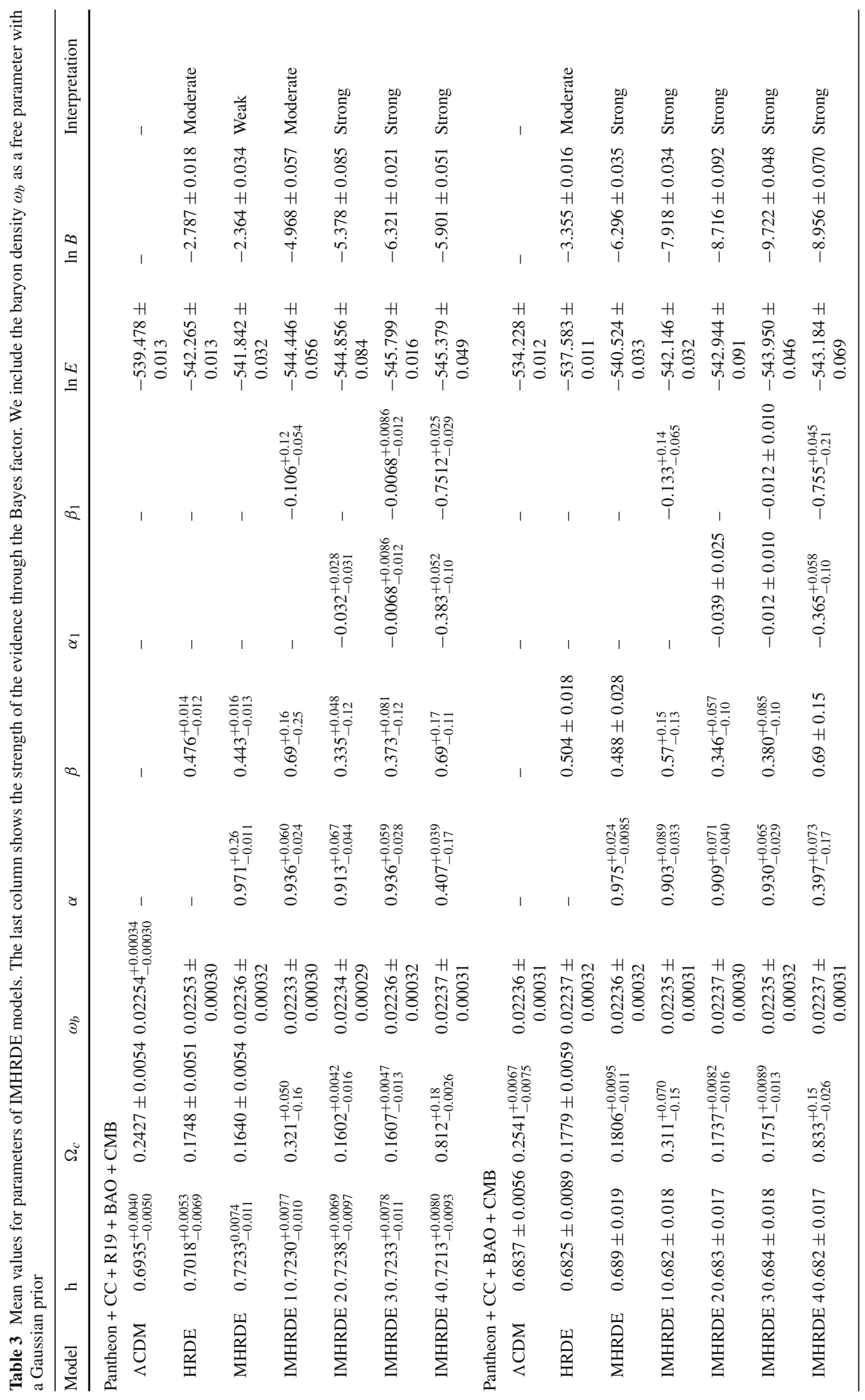


for the $\Lambda \mathrm{CDM}$ model. However, in properly studying the $H_{0}$ tension we should consider the full anisotropy spectrum of CMB data (not just one data as in our case) and should not consider the R19 data in the analysis [102]. In this sense, considering the analysis without the R19 data we notice that the value of the $h$ parameter is closer to the Planck value and there is not substantial difference in the resulting value for $\Lambda \mathrm{CDM}$ or the IMHRDE models.

As a comparison, in Tables 2 and 3, we also indicate the results for models HRDE and MHRDE (without interaction). For these scenarios in the full joint analysis, Pantheon $+\mathrm{CC}+$ $\mathrm{R} 19+\mathrm{BAO}+\mathrm{CMB}$, evidence indicates more support when compare to the interacting ones, nevertheless the evidence also disfavor these models when compare to $\Lambda$ CDM.

In the literature there are several studies analyzing the performance of holographic dark energy models in fitting background data compared to $\Lambda$ CDM. In particular, in Ref. [36] the holographic Ricci dark energy model (HRDE) without interaction is analyzed, the authors find evidence disfavoring this model when compared to $\Lambda$ CDM. In Ref. [40] interacting HRDE is studied with the AIC and BIC criteria and the interacting HRDE model is concluded ruled out. In Ref. [46] a modified holographic Ricci dark energy model without interaction is considered and the $\chi^{2} /$ dof criteria is used to reach the same conclusion. In Ref. [70] many interacting HRDE models are studied and all of them are discarded according to the BIC criteria when compared to $\Lambda$ CDM. In Ref. [72] HRDE and MHRDE models are analyzed without interaction, beyond background data the authors consider growth rate data, with AIC and BIC the authors find strong indications against holographic models when compared to $\Lambda \mathrm{CDM}$. Likewise, in our work we find strong evidence against the linear interacting modified holographic Ricci dark energy models studied (see Tables 2 and 3 ) when compared to $\Lambda$ CDM in light of the most recent background data.

In Figs. 1, 2, 3, and 4 we show the contours of $68.3 \%$ and $95.4 \%$ confidence levels for the full joint analysis (Table 2), corresponding to IMHRDE 1, 2, 3, 4, respectively. Notice that the posteriors for the parameter space in models IMHRDE 1 and 4 result to be multi-modal, nevertheless, the Multinest code is designed to deal with this issue, not representing a problem in computing the Bayesian evidence [97].

Finally, from Eq. (19) we can evaluate the performance of the IMHRDE models in alleviating the coincidence problem. By considering the mean values for the parameters in the full joint analysis (Table 2) we notice that IMHRDE 1, 3 and 4 alleviate the coincidence problem (the coincidence parameter tends to a positive constant in the future). For models HRDE, MHRDE and IMHRDE 2 the coincidence problem is not alleviated.

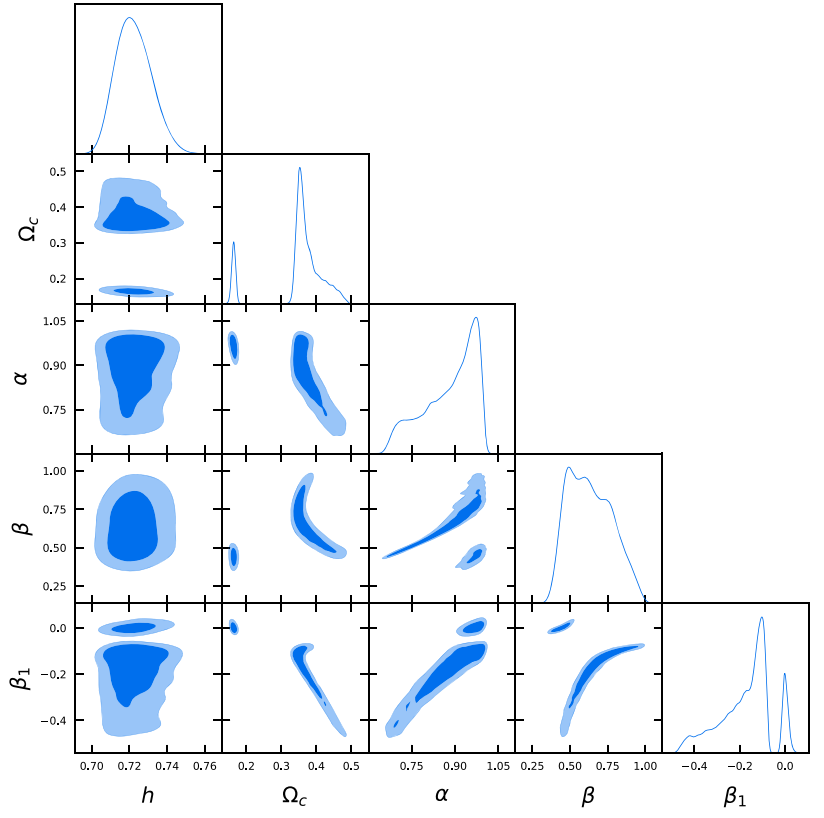

Fig. 1 Contour plots for model IMHRDE1 showing the $1 \sigma$ and $2 \sigma$ regions. We considered the full joint analysis with Pantheon $+\mathrm{CC}+$ $\mathrm{R} 19+\mathrm{BAO}+\mathrm{CMB}$

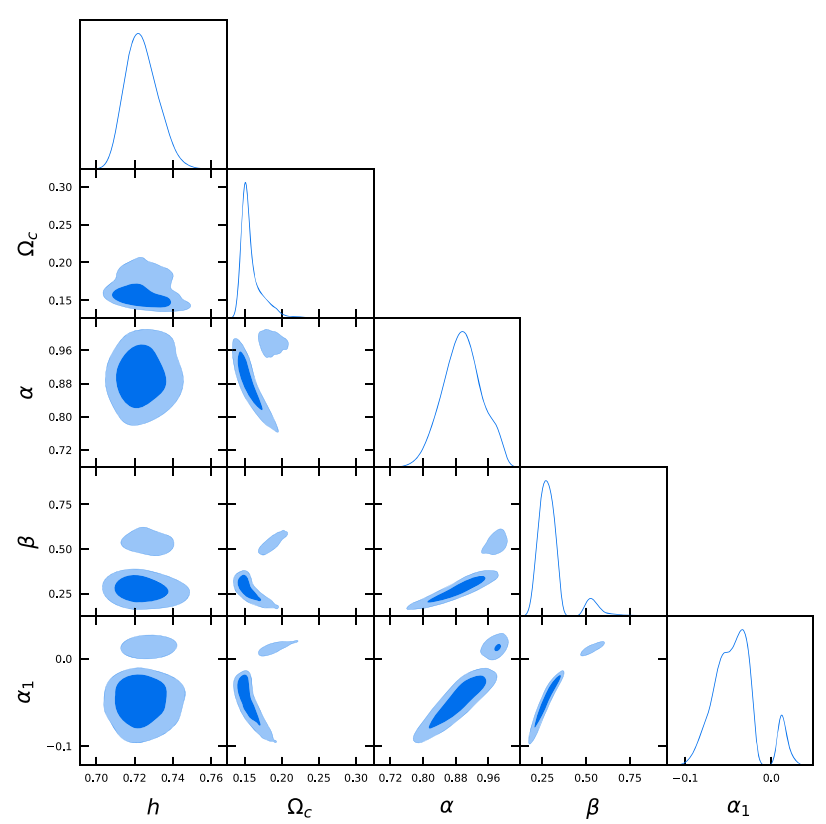

Fig. 2 Contour plots for model IMHRDE2 with the $1 \sigma$ and $2 \sigma$ regions. We considered the full joint analysis with Pantheon $+\mathrm{CC}+\mathrm{R} 19+\mathrm{BAO}$ $+\mathrm{CMB}$

\section{Final remarks}

In this work we have studied interacting modified holographic Ricci dark energy models, where linear interactions are considered. We have found analytical solutions to these scenarios (see Eq. (16)) and we have performed a Bayesian model selection analysis. The Bayesian comparison is per- 


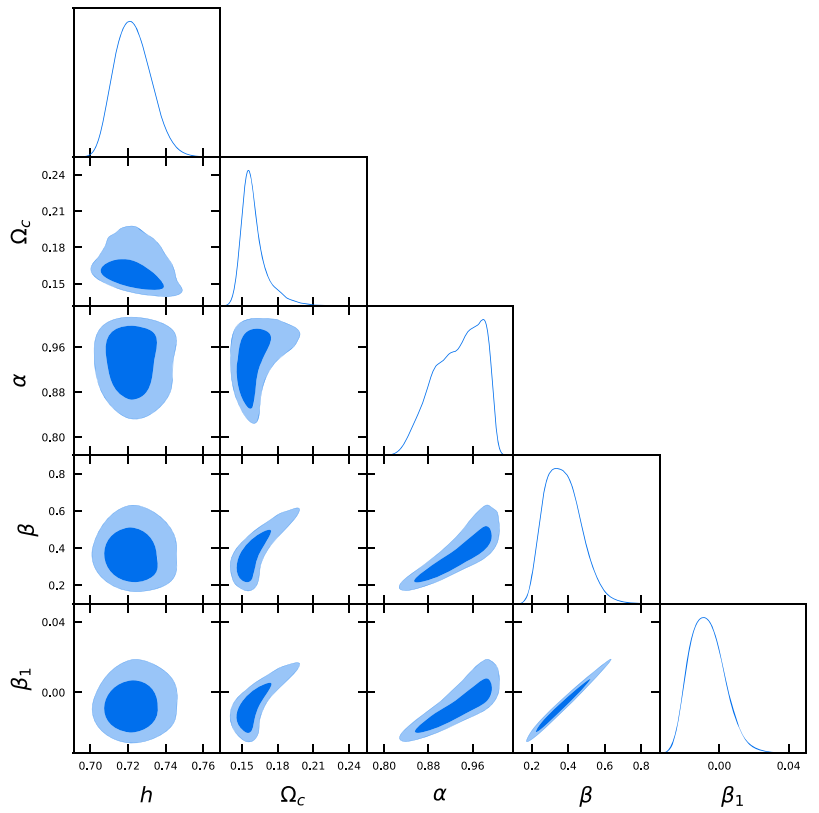

Fig. 3 Contour plots for model IMHRDE3 with the $1 \sigma$ and $2 \sigma$ regions. We considered the full joint analysis with Pantheon $+\mathrm{CC}+\mathrm{R} 19+\mathrm{BAO}$ $+\mathrm{CMB}$

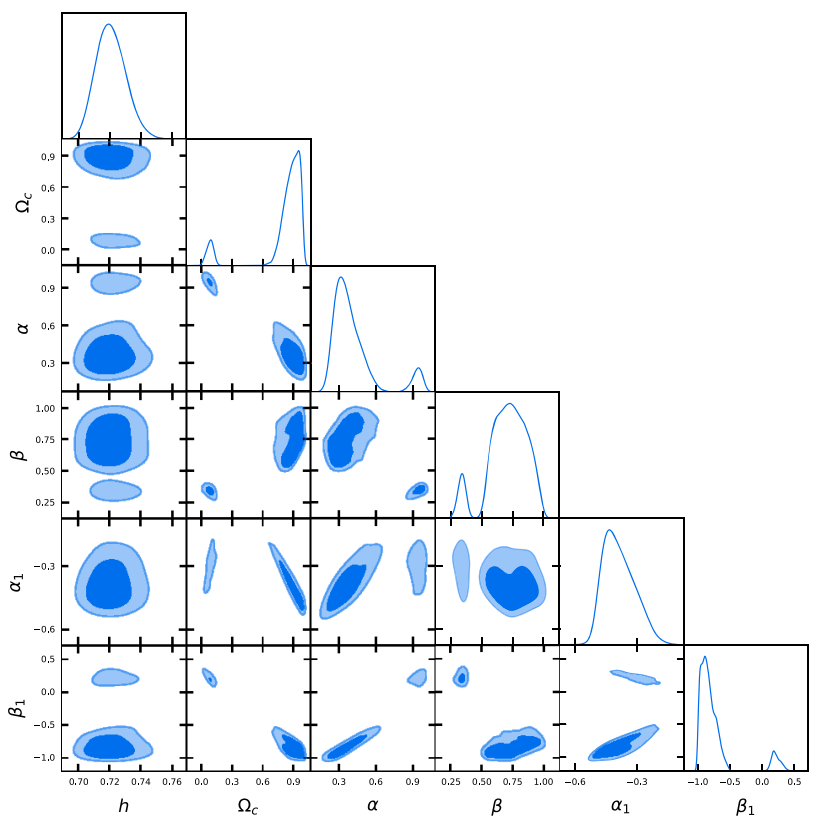

Fig. 4 Contour plots for model IMHRDE4 with the $1 \sigma$ and $2 \sigma$ regions. We considered the full joint analysis with Pantheon $+\mathrm{CC}+\mathrm{R} 19+\mathrm{BAO}$ $+\mathrm{CMB}$

formed with three different combinations of background data, Pantheon + R19, Pantheon + CC + R19+ BAO + CMB and Pantheon $+\mathrm{CC}+\mathrm{BAO}+\mathrm{CMB}$ (see Sect. 3), and the fiducial model is assumed to be $\Lambda \mathrm{CDM}$. Our results indicate that, with the full joint analysis including or not R19, there is moderate/strong evidence against the IMHRDE models studied, this conclusion is consistent with several studies where holographic dark energy models have been contrasted with background data $[36,40,46,53,70-72]$. We also noticed that the baryon density parameter $\omega_{b}$ have minimal impact in our overall results. On the other hand, the $H_{0}$ tension can not be addressed with the present analysis because we have not included the full anisotropy spectrum of CMB, which originates the tension. Finally, the present analysis is performed at background level, a profound analysis considering perturbations to the models could, besides of addressing the $H_{0}$ tension, identify instability regions which could help to restrict the parameter space prior distributions.

Acknowledgements AC was partially supported by Dirección de Investigación Universidad del Bío-Bío through grant no. GI-172309/C. CRB thanks the financial support of Dirección de Postgrado through "Beca de Investigación de Postgrado 2018" and Dirección de Investigación Universidad del Bío-Bío through grants no. GI-172309/C and DIUBB 181907 4/R.

Data Availability Statement This manuscript has no associated data or the data will not be deposited. [Authors' comment: The data used in this paper are publicly available, the datasets used are referenced in the paper.]

Open Access This article is licensed under a Creative Commons Attribution 4.0 International License, which permits use, sharing, adaptation, distribution and reproduction in any medium or format, as long as you give appropriate credit to the original author(s) and the source, provide a link to the Creative Commons licence, and indicate if changes were made. The images or other third party material in this article are included in the article's Creative Commons licence, unless indicated otherwise in a credit line to the material. If material is not included in the article's Creative Commons licence and your intended use is not permitted by statutory regulation or exceeds the permitted use, you will need to obtain permission directly from the copyright holder. To view a copy of this licence, visit http://creativecomm ons.org/licenses/by/4.0/.

Funded by SCOAP $^{3}$.

\section{References}

1. D.H. Weinberg, M.J. Mortonson, D.J. Eisenstein, C. Hirata, A.G. Riess, E. Rozo, Phys. Rept. 530, 87-255 (2013)

2. S. Weinberg, Rev. Mod. Phys. 61, 1-23 (1989)

3. S. Weinberg, (2000). arXiv:astro-ph/0005265

4. L.P. Chimento, A.S. Jakubi, D. Pavon, W. Zimdahl, Phys. Rev. D 67, 083513 (2003)

5. Z.K. Guo, Y.Z. Zhang, Phys. Rev. D 71, 023501 (2005)

6. E. Di Valentino, A. Melchiorri, O. Mena, Phys. Rev. D 96(4), 043503 (2017)

7. E. Di Valentino, A. Melchiorri, O. Mena, S. Vagnozzi, Phys. Dark Univ. 30, 100666 (2020)

8. S. Pan, W. Yang, E. Di Valentino, E.N. Saridakis, S. Chakraborty, Phys. Rev. D 100(10), 103520 (2019)

9. K.L. Pandey, T. Karwal, S. Das, JCAP 07, 026 (2020)

10. M. Benetti, W. Miranda, H.A. Borges, C. Pigozzo, S. Carneiro, J.S. Alcaniz, JCAP 12, 023 (2019)

11. J. Solà, A. Gómez-Valent, J. de Cruz Pérez, Phys. Lett. B 774, 317 (2017)

12. D. Pavon, W. Zimdahl, Phys. Lett. B 628, 206-210 (2005)

13. L. Verde, T. Treu, A.G. Riess, Nat. Astron. 3, 891 (2019)

14. A.G. Riess et al., Astrophys. J. 826(1), 56 (2016) 
15. A.G. Riess et al., Astrophys. J. 730, 119 (2011) [Erratum: Astrophys. J. 732, 129 (2011)]

16. E.J. Copeland, M. Sami, S. Tsujikawa, Int. J. Mod. Phys. D 15, 1753-1936 (2006)

17. J. Yoo, Y. Watanabe, Int. J. Mod. Phys. D 21, 1230002 (2012)

18. S. Wang, Y. Wang, M. Li, Phys. Rept. 696, 1-57 (2017)

19. S. Bahamonde, C.G. Böhmer, S. Carloni, E.J. Copeland, W. Fang, N. Tamanini, Phys. Rept. 775-777, 1-122 (2018)

20. G. 't Hooft, Conf. Proc. C 930308, 284-296 (1993)

21. J.D. Bekenstein, Phys. Rev. D 7, 2333-2346 (1973)

22. J.D. Bekenstein, Phys. Rev. D 9, 3292-3300 (1974)

23. J.D. Bekenstein, Phys. Rev. D 23, 287 (1981)

24. J.D. Bekenstein, Phys. Rev. D 49, 1912-1921 (1994)

25. S.W. Hawking, Phys. Rev. D 13, 191-197 (1976)

26. S.W. Hawking, Commun. Math. Phys. 43, 199-220 (1975) [Erratum: Commun. Math. Phys. 46, 206 (1976)]

27. A.G. Cohen, D.B. Kaplan, A.E. Nelson, Phys. Rev. Lett. 82, 49714974 (1999)

28. S.D. Hsu, Phys. Lett. B 594, 13-16 (2004)

29. M. Li, Phys. Lett. B 603, 1 (2004)

30. R.G. Cai, Phys. Lett. B 657, 228-231 (2007)

31. C. Gao, F. Wu, X. Chen, Y.G. Shen, Phys. Rev. D 79, 043511 (2009)

32. R.G. Cai, B. Hu, Y. Zhang, Commun. Theor. Phys. 51, 954-960 (2009)

33. L. Xu, W. Li, J. Lu, Mod. Phys. Lett. A 24, 1355-1360 (2009)

34. C.J. Feng, Phys. Lett. B 672, 94-97 (2009)

35. C.J. Feng, Phys. Lett. B 670, 231-234 (2008)

36. M. Li, X.D. Li, S. Wang, X. Zhang, JCAP 06, 036 (2009)

37. X. Zhang, Phys. Rev. D 79, 103509 (2009)

38. S. Lepe, F. Pena, Eur. Phys. J. C 69, 575-579 (2010)

39. K.Y. Kim, H.W. Lee, Y.S. Myung, Gen. Relativ. Gravity 43, 1095$1101(2011)$

40. S. del Campo, J. Fabris, R. Herrera, W. Zimdahl, Phys. Rev. D 83, 123006 (2011)

41. S. del Campo, J.C. Fabris, R. Herrera, W. Zimdahl, Phys. Rev. D 87(12), 123002 (2013)

42. L. Granda, A. Oliveros, Phys. Lett. B 669, 275-277 (2008)

43. L. Granda, A. Oliveros, Phys. Lett. B 671, 199-202 (2009)

44. L. Granda, A. Oliveros, (2009). arXiv:0901.0561 [hep-th]

45. L. Granda, W. Cardona, A. Oliveros, (2009). arXiv:0910.0778 [hep-th]

46. Y. Wang, L. Xu, Phys. Rev. D 81, 083523 (2010)

47. T.K. Mathew, J. Suresh, D. Divakaran, Int. J. Mod. Phys. D 22, 1350056 (2013)

48. M. Tavayef, A. Sheykhi, K. Bamba, H. Moradpour, Phys. Lett. B 781, 195-200 (2018)

49. H. Moradpour, S.A. Moosavi, I.P. Lobo, J.P. Morais Graça, A. Jawad , I.G. Salako, Eur. Phys. J. C 78(10), 829 (2018)

50. A. Sayahian Jahromi, S.A. Moosavi, H. Moradpour, J.P. Morais Graça, I.P. Lobo, I.G. Salako, A. Jawad, Phys. Lett. B 780, 21-24 (2018)

51. E.N. Saridakis, Phys. Rev. D 102, 123525 (2020)

52. B. Hu, Y. Ling, Phys. Rev. D 73, 123510 (2006)

53. T.F. Fu, J.F. Zhang, J.Q. Chen, X. Zhang, Eur. Phys. J. C 72, 1932 (2012)

54. L.P. Chimento, M.I. Forte, M.G. Richarte, Mod. Phys. Lett. A 28, 1250235 (2013)

55. L.P. Chimento, M.G. Richarte, Phys. Rev. D 84, 123507 (2011)

56. L.P. Chimento, M.G. Richarte, Phys. Rev. D 85, 127301 (2012)

57. L.P. Chimento, M.I. Forte, M.G. Richarte, AIP Conf. Proc. 1471, $39(2012)$

58. L.P. Chimento, M. Forte, M.G. Richarte, Eur. Phys. J. C 73(1), 2285 (2013)

59. P. Pankunni, T.K. Mathew, Int. J. Mod. Phys. D 23, 1450024 (2014)
60. F. Arevalo, P. Cifuentes, S. Lepe, F. Peña, Astrophys. Space Sci. 352, 899-907 (2014)

61. L.P. Chimento, M.G. Richarte, Eur. Phys. J. C 73(4), 2352 (2013)

62. A. Oliveros, M.A. Acero, Astrophys. Space Sci. 357(1), 12 (2015)

63. S. Som, A. Sil, Astrophys. Space Sci. 352, 867-875 (2014)

64. N. Mahata, S. Chakraborty, Mod. Phys. Lett. A 30(27), 1550134 (2015)

65. S. Pan, S. Chakraborty, Int. J. Mod. Phys. D 23(11), 1450092 (2014)

66. F. Arevalo, P. Cifuentes, F. Pena, Astrophys. Space Sci. 361(1), 45 (2016)

67. S. Lepe, F. Peña, Eur. Phys. J. C 76(9), 507 (2016)

68. M.A. Zadeh, A. Sheykhi, H. Moradpour, Int. J. Mod. Phys. D 26(08), 1750080 (2017)

69. R. Herrera, W. Hipolito-Ricaldi, N. Videla, JCAP 08, 065 (2016)

70. L. Feng, X. Zhang, JCAP 08, 072 (2016)

71. P. George, T.K. Mathew, (2019). arXiv:1906.08532 [gr-qc]

72. I. Akhlaghi, M. Malekjani, S. Basilakos, H. Haghi, Mon. Not. R. Astron. Soc. 477(3), 3659-3671 (2018)

73. R. D'Agostino, Phys. Rev. D 99(10), 103524 (2019)

74. F. Arevalo, A. Cid, J. Moya, Eur. Phys. J. C 77(8), 565 (2017)

75. B. Santos, N.C. Devi, J. Alcaniz, Phys. Rev. D 95(12), 123514 (2017)

76. A. Heavens, Y. Fantaye, E. Sellentin, H. Eggers, Z. Hosenie, S. Kroon, A. Mootoovaloo, Phys. Rev. Lett. 119(10), 101301 (2017)

77. S. Santos da Costa, M. Benetti, J. Alcaniz, JCAP 03, 004 (2018)

78. U. Andrade, C. Bengaly, J. Alcaniz, B. Santos, Phys. Rev. D 97(8), 083518 (2018)

79. T. Ferreira, C. Pigozzo, S. Carneiro, J. Alcaniz, (2017). arXiv:1712.05428 [astro-ph.CO]

80. A. Cid, B. Santos, C. Pigozzo, T. Ferreira, J. Alcaniz, JCAP 03, $030(2019)$

81. D.M. Scolnic et al., Astrophys. J. 859(2), 101 (2018)

82. M. Moresco et al., JCAP 1605(05), 014 (2016)

83. A.G. Riess, S. Casertano, W. Yuan, L.M. Macri, D. Scolnic, Astrophys. J. 876(1), 85 (2019)

84. F. Beutler et al., Mon. Not. R. Astron. Soc. 416, 3017 (2011)

85. A.J. Ross, L. Samushia, C. Howlett, W.J. Percival, A. Burden, M. Manera, Mon. Not. R. Astron. Soc. 449(1), 835 (2015)

86. M. Ata et al., Mon. Not. R. Astron. Soc. 473(4), 4773 (2018)

87. J. Hou et al., Mon. Not. R. Astron. Soc. 480(2), 2521 (2018)

88. S. Alam et al. [BOSS Collaboration], Mon. Not. R. Astron. Soc. 470(3), 2617 (2017)

89. P.A.R. Ade et al. [Planck Collaboration], Astron. Astrophys. 594, A14 (2016)

90. R. Jimenez, A. Loeb, Astrophys. J. 573, 37 (2002)

91. L. Verde, P. Protopapas, R. Jimenez, Phys. Dark Univ. 5-6, 307 (2014)

92. N. Aghanim et al. [Planck], Astron. Astrophys. 641, A6 (2020)

93. D.J. Eisenstein et al. [SDSS Collaboration], Astrophys. J. 633, 560 (2005)

94. D.J. Eisenstein, W. Hu, Astrophys. J. 496, 605 (1998)

95. J. Evslin, A.A. Sen, Ruchika, Phys. Rev. D 97(10), 103511 (2018)

96. R. Trotta, Contemp. Phys. 49, 71 (2008)

97. F. Feroz, M. Hobson, Mon. Not. R. Astron. Soc. 384, 449 (2008)

98. F. Feroz, M. Hobson, M. Bridges, Mon. Not. R. Astron. Soc. 398, 1601-1614 (2009)

99. R.J. Cooke, M. Pettini, C.C. Steidel, Astrophys. J. 855(2), 102 (2018)

100. J. Lesgourgues, G. Mangano, G. Miele, S. Pastor, Neutrino Cosmology (Cambridge University Press, Cambridge, 2013)

101. E. Komatsu et al. [WMAP Collaboration], Astrophys. J. Suppl. 192, 18 (2011)

102. E. Di Valentino, A. Melchiorri, O. Mena, S. Vagnozzi, Phys. Rev. D 101(6), 063502 (2020) 